\title{
QUI FAIT ET DÉFAIT LES GOUVERNEMENTS
}

\author{
François Perin
}

CRISP | «Courrier hebdomadaire du CRISP »

$1958 / 0 \mathrm{n}^{\circ} 0 \mid$ pages XV à XX

ISSN 0008-9664

Article disponible en ligne à l'adresse :

https://www.cairn.info/revue-courrier-hebdomadaire-du-crisp-1958-0-page-XV.htm

Distribution électronique Cairn.info pour CRISP.

(C) CRISP. Tous droits réservés pour tous pays.

La reproduction ou représentation de cet article, notamment par photocopie, n'est autorisée que dans les limites des conditions générales d'utilisation du site ou, le cas échéant, des conditions générales de la licence souscrite par votre établissement. Toute autre reproduction ou représentation, en tout ou partie, sous quelque forme et de quelque manière que ce soit, est interdite sauf accord préalable et écrit de l'éditeur, en dehors des cas prévus par la législation en vigueur en France. Il est précisé que son stockage dans une base de données est également interdit. 
Qui fait et défait les gouvernements.

En I958, la Belgique a connu trois gouvernements différents. Tous les départements ont eu au moins deux titulaires mais le Commerce Extérieur, les Colonies, I'Instruction Publique, l'Intérieur, la Justice, la Senté Publique et la Familie, en ont ou trois. In I958, le département des Affaires Bconomiques a été géré par quatre ministres différents.

De I9I8 à I958, la Belgique a connu 23 crises gouvernementeles de caractere politique ('à l'exclusion de celles qui surviennent - de caractère constitutionnel - à chaque élection législative).

Le phénomène des crises gouvernementales a donc sérieusement marqué le déroulement de la vie politique belge. Comment se forment les gouvernements ? Quels sont les causes et les mécanismes des crises ministrielles ? Telles sont les deux questions étudiées ici par F: PRRIN. I'étude couvre la période I9I8-I958.

La formation du gouvernement.

Le Ro1 ne peut constituer un gouvernement sans que celul-ci n'obtienne, immédiatement après la nomination des ministres, la confience des chambres par un vote sur une résolution expressément formulée.

Le Roi est done tenu de former un gouvernement susceptible de recuellIir la confiance des chambres. Son choix n'est pas personnel. La composition politique des chambres constitue en droit constitutionnel un élément légitimement déterminant ( I).

Le Roi apprécie-t-il seul la situation politique ? Evidemnent non ; le monarque procède à des consultations devenues traditionnelles. Il consulte le président de la Chambre et celul du Sénat qui peuvent, en raison même de leur fonction, apprécier les chances d'une formule gouvernementale devent les assemblées dont ils dirigent les débats.

( I ) $\nabla$. Le chef de I'Itat et le gouvernement, W.J. Ganshof van der Meersch Revue de droit international et de droit comparé I950, p. I88. 
Le Rol consulte aussi le Premier Ministre démissionnaire, les leaders des groupes parlementaires, notament ceux de l'opposition, les chels de partis, les homes les plus représentatifs de l'opinion.

La formation du gouvernement se fait en deux ou trols temps : le Rol désigne parfois directement le Premier Ministre, celui-cl compose ensuite la liste des ministres et la soumet au Ro1. Parfois, avant de désigner le "formateur" du gouvernement, le Roi, dans les cas diffielles, désigne un "Informateur" ( 2 ) qui se substitue à lui pour procéder à des consultations politiques et qui lui falt rapport sur la situation.

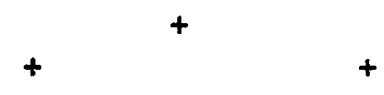

Corment le choix du Roi est-il finalement déterminé ? Quelles sont les interventions décisives?

Aucun parti ne réunissant la majorité absolue depuis I9I8 ( 3 ), la tâche du Rol est peu alsée; il ne lui suffit pas, comme en Angleterre, de se tourner vers le leader du parti le plus pulssant.

Le Lait merquant de la pérlode qui nous ocoupe est l'intervention directe des états-majors des partís politiques. On sait que ceux-ci se réunissent en congres, organe souverain de chaque organisation politique; formé des délégués des diverses sections locales; ces congres él1sent des bureaur ou comités centraux qui prennent une part actire à la formation des gourernements. Leur action est plus ou moins discréte. Aucun parti n'a jamais été jusqu'à voter une résolution rendue publique pour désigner formellement un candidat premier minlstre.

Les partis ont un minimum de respect pour le formalisme constitutlonnel mais 11 est notoire que oertains premiers minlstres ont été délibérément choisis par leur propre part1 (4).

Ia plupart du temps, lo choix de la personne du premler ministro résulte des négociations entamées entre les personnalités politiques oonsultée's par lo chef de l'Itat.

Le premier ministre doit être non seulement agréé par son propre perti mais égelement par los partis qui feront partio de la coalition. C'est alnsi que M. Poullet a pu étre présenté comme premier ministre apres les électlons du 5 avrll I925 parce qu'1l étalt la personnalite cathollque qui menifestalt lo plus de compréhension et de sympathie à l'égard du Part1 Ouvrier Belge qui allait entrer dans une coalition catholique-socialiste. Il en est de même de M. P.H. Spaek, lors de la formation du gouvernement catholique-socialiste le 20 mars I947 et de M. Hyskens, chef de la coelition catholique-11beralo du II sout I949.

(2) Voyez notament la désignation de M. Theunis, le 22 mars I935, M. Tschoffen, Ie 22 septembre I944, M. de Schryver, après les élections du I7 février I946 et du Ier Juin I958.

( 3 ) Sauf lo pert1 soclal-chrétien de I950 à I954.

(4) C'est le ces notamment de M. Achille Van Aoker ( lo I2 février I945, le 3I mars I946 et le 23 arril I954) de M. Huysmans ( 3 août I946) et de M. Van Houtte (I5 jenv1er I952). M. Hyskens ( 4 juln I958). 
La personne du premier ministre s'impose parfois par son attitude politique pendant la vie du gouvernement précédent. C $C^{9} e s t$ ainsi qu'après la chute du gouvernement Poullet-Vandervelde en I926, le Rol désigna M. Jaspar comme premier ministre; M. Jaspar était précisément le leader conservateur qui avait dirigé l'opposition contre le gouvernement Poullet. Cette manière de voir est même considérée comme un principe fondamental du régime parlementaire. Le Parti Ouvrier Belge fit au Rol un vif reproche de ne pes l'avoir appliqué après la crise du 27 février 1924. Le gouvernement avait été renversé à la Chambre par le vote conjugué des socialistes et des catholiques flamands. Ie Roi fut vivement attaqué pour n'avoir pas essayé de constituer un gouvernement à l'image de cette opposition (I)。

Il arrive que des milieux distinds du monde politique jouent un róle important dans la désignation du premier ministre. Les milieux financiers et bancaires $n^{\prime} y$ sont pas étrangers ( 2 ).

Quelle est la part de I'influence personnelle du Roi ? Il est extrêmement difficlle de répondre à cette question. L'action du monarque est couverte per une discrétion traditionnelle résultant du principe constitutionnel de l'irresponsabilité royale. Il est donc impossible, faute de documents, de dégager avec précision le róle personnel de la monarchle. II paraft certain que le Roi ne peut faire de choix exclusivement personnel. Il ne peut donner libre cours à ses préférences politiques. Aucune désignation n'est le fait de la Cour ( 3 ). Mais un Roi habile, dans les moments difficiles, peut certes orienter les négoclations et faire surgir une solution qu'il appelle personnellement de ses voeux $(4)$.

Par exemple, il semble bien que le choix de M. Carton de wiart le 7 notembre I920 résulte vraisemblablement d'un idée personnelle du Roi Albert, encore que celui-ci att commencé par pressentir M. Segers qui déclina l'offre de former le gouvernement. Il en est de même de la personno de $\mathrm{M}$. de Broqueville, premier ministre d'octobre I932 à novembre I934; cet homme politique jouissait de toute la sympathie du Roi Albert $(5)$.

Par contre, il n'est pas impossible que le Roi écarte des personnalltés qui n'ont pas sa confiance. Il n'est pas rare non plus que le Roi manifeste publiquement ses préférences en pressentant une personnalité déterminée qui décline d'ailleurs cette invitation parce qu'elle ne correspond ni à ses vues ni à celles de son parti. Ainsi, le I2 avril I939, le Roi Léopold offrit à $M$. Jaspar le soin de former le gouvernement et au mois d'avril I954, le Roi Baudouin fit à M. Max Buset la même offre. Cette offre ne peut être interprétée comme un geste purement protocolaire.

( I ) Résolution du Conseil général du P.O.B. du 4 mars I924, A.P. Ch. , I9.3.I924, p. 673 et s, et 683 et s., intervention de MM. Hubin et Destrée.

(2) Voyez notamment la désignation de M. Delacrolx le 22 novembre I9I8, de M. Theunis, le 5 décembre I92I et le I6 novembre I934, de M. van Zeeland, le 23 mars 1935.

(3) Sauf peut-être M. Pholien le I6 août I950, après l'effacement de Léopold III, et encore, les réactions internes du P.S.C. n'y sont pas étrangères.

( 4 ) Voyez l'attitude du Roi Albert entre le 27 fétrier et le 6 mars I924 lors du choix du premier ministre Theunis ( Höjer, op. cit., p. I40).

( 5 ) Höjer, op.cit.p.208. 
Cette influence active ne s'exerce qu'à l'égard du premier ministre. Une fois le " formateur " pressenti, c'est lui qui dresse la liste de ses coéquipiers. Cette liste doit être soumise au Rol quil ne peut plus intervenir que sous forme d'exclusives discrètes, celles-ci n'aboutissant d'ailleurs à écarter un candidat que dans la mesure où le formateur n'a pas tenu lui-même à Ie consacrer.

Notons, toutefo1s, qu'il est arrivé au Ro1 de persuader personnellement un candidat hésitant d'entrer dans un ministère ( I ).

Pour la composition du gouvernement, y compris le oholx des personnes, l'intervention des pertis est fréquente et déterminante. Fille n'est pas exclusive, d'autres influences se font souvent sentir.

Ains1, le premier gouvernement formé par le Rol Albert après I'armistice du II notembre I9I8, présidé par M. Delacroix ( lo 22 novembre I9I8), trouve son orlgine dans les négociations poursuivies au chêteau de Lophem entre le Rol et certains hommes politiques. Ie comité national de secours et d'alimentation, dirlgé par lo financier Francqui, exerça une forte influence our la formation du gouvernement $(2)$. Ce comité de pur fait avait acquis pendant la guerre un prestige considérable en pays occupé, tent par son action que par la personnalité de ses membres. M. Delacrolx en falsait d'allleurs partie.

I'intervention des partis sur le programe comme sur le cholx des personnes fut évidente ( 3 ). Les futurs ministres socialistes furent nentis d'une autorisation de leur pert1 exprimée per un vote du Consell général (4).

Le deuxième gouvernement présidé per M. Deleorolx fut influenoé de la même maniere par les partis ( 5 )

Les gouvernements formés par M. Certon de Wiart le 23 novembro I920 ( 6 ), M. Poullet-Vendervelde le 23 juin I925, Jespar lo 20 mal I926 ( 6 ), Renkin lo 5 juin I93I, De Broqueville lo 22 octobre I932, ot le I7 décembre I932, ven Zeeland I0 I2 décembre I935, Plerlot lo In avil I939 rósulterent de négooiatlons parfols longues et difficlies entre personnelités politiques, plus ou molns formellement mandatées par leurs groupes politiques ou certains organes de lours pertis.

I'Intervention du Conseil national du parti liberal d'une part, du Congrès, ou du Consell général du P.O.B. d'autre part, est à pou près constante: du côté catholique, celle de la Fédération des corclos et le la Ilgue nationalo des travallleurs ohrétions n'est pas rare.

( I ) ex. : M; Hubert dens lo gouvernement Theunis, V. 2e presse des I3 ot I4 décembre IORI, MM. Hymans, Devèze, Jenson, Forthome, lo Is décembre I932. H6jor, 0p. 01t. p. 208 - $2 I 2$.

(2) Sur la crise de Lophom, rolr C.H. Hbjor, Le régime parlementaire belge, p. 63 a 90 . Sur lo Consell national, 1dem, p. 79.

(3) V. résolution des gauches libérales du I6 ootobre I9I8, résolution du consell générel du P.O.B. du 30 ootobre I9I8 et du I8 novembre - Hojer, p. 79 et 87.

(4) V. HOjer, p. IOZ-IO3.

(5) Laem, p. IIZ.

(6) I'Intervention du Ro1 fut partioulièrement aetive au cours des négoolations. Le cabinet du Rol publia des communiqués le Is et le 22 mal I926. 
Après I945, I'intervention des partis est.régulière mais elle a donné lieu à beaucoup moins de difficultés qu'entre I9I8 et I939.

La situation politique qui mit le plus de temps à se dénouer est celle qui résulta des élections de février I946. En dehors de ce cas, les gouvernements se constituèrent assez aisément. Le róle de la monarchie fut donc infiniment plus effacé qu'entre les deux guerres.

I'intervention des milieux financiers, dont certaines personnalités sont directement consultées par le Roi ou par les hommes politiques, est particulièrement marquante dans la formation des gouvernements présidés par MM. Delacroix ( 22 novembre I9I8), Theunis ( Ie I6 décembre I92I), Jaspar ( le 20 mai I926), Theunis ( 1 e 20 novembre I934) et van Zeeland ( Ie 25 mai I935). Lors de la formation de ce dernier gouvernement, le Roi, pour la première fois, consulta également les représentants des grandes organisations économiques et syndicales.

L'intervention des milieux bancaires est moins ostensible après I945 mais il faut néanmoins faire observer que deux ministres des finances furent des banquiers ( I ).

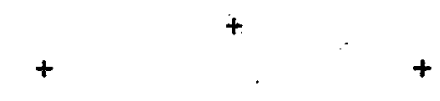

\section{Comment s'élabore la composition interne des gouvernements?}

Le nombre de ministres d'une tendance politique déterminée est-il proportionnel à la force numérique de cette tendance au parlement ? Un calcul proportionnel purement arithmétique est impossible; si une certaine proportion est respectée en ce qui concerne les catholiques et les socialistes, on peut constater que la proportion des ministres libéraux est constamment surévaluée par rapport à la force parlementaire de leur part1. Ce phénomène est dû, croyonsnous, à la position centrale de ce parti dont l'appul est constamment recherché par les éventuels partenaires gouvernementaux. On a reproché aux libéraux leur goût disproportionné pour les portefeuilles ministériels.

Mais l'histoire parlementaire prouve qu'ils sont constament sollicités par les autres partis ou par la couronne elle-même. L'absence de majorité absolue et la répugnance naturelle que catholiques et socialistes ont eu à s'associer, ont fait des libéraux des partenaires indispensables. Les conservateurs catholiques s'inquiètent à l'fáé de laisser les libéraux dans l'opposition, leur tendance économique et sociale étant fort souvent la même que la leur. Le parti socialiste dont la tendance radicale est manifeste, surtout en Wallonie, répugne également, pour d'autres motifs, à laisser les Iibéraux en dehors des responsabilités ministérielles. Ils craignent que le sentiment anticlérical de leur clientèle électorale ne solt exploité par lo parti libéral, alors qu'ils sont liés par une alliance gouvernementale avec le parti, qu'ils qualifient aux-mêmes péjorativement de clérical. Ceci explique peut-être la raison pour laquelle, sur 40 ans de régime de suffrage unịversel, les libéraux totalisent 32 années de participation ministérielle.

( I) MN. Gutt et A.E. Janssen. Th outre, il faut citer les ministres De Voghel ( Banque Nationale) et J. Vauthier ( Iiquidateur d'organismes financiers issus de la guerre, occupent actuellement une fonction importante à la Banque de Bruxelles.) 
Le dosage des portefeuilles ministériels n'est pas seulement déterminé par l'appartenance à un parti politique. La dualité ethnique du pays, les mouvements flamands et wallons ont fortement influencé la composition de tous les gouvernements. Enfin, les classes sociales différentes exigent une représentation spéciale ( I ). Les agriculteurs, les classes moyennes, les milieux syndicaux, sont perticulièrement susceptibles à cet égard.

La compétence personnelle joue parfois un róle déterminant ; la préférence de M. Hymans et M. P.H. Spaak pour les affaires étrangères est évidente. Celle de M. Lefebrre, ministre de I'agriculture en I946 et dë I954 à I958, se justifie par une activité essentiellement orientée vers l'agriculture ( 2 ), l'Inclination de M. Plerre Vermeylen pour le département de 1 'intérieur ( I947I949 - I954- I958) n'est pas non plus l'effet d'un hasard ( 3 ). La défense nationale a vu plusieurs offieiers supérieurs investis de la fonction de ministre. Nous avons signalé à plusieurs reprises la présence de banquiers ou de financiers à la tôte du département des finances. Mais, par ailleurs, règne l'indétermination la plus complète.

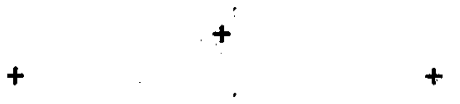

Le principe même de l'intervention des partis a été exprimé formellement à maintes reprises.

C'est ainsi que le Congrès libéral des I6-I8 octobre I920, dans sa résolution déclarait que "l'adhásion définitivo de mandataires libéraux à un cabinet en formation sera subordonnée à la ratification préalable par le congrès libéral des bases politiques, économique et sociale sur lesquelles le ministere entendra se constituér " .

D'autre part, on peut IIre dans "Ie Peuple " du 8 juin I925, lors de la formation du gouvernement Poullet-Vandervelde, que " le Consell général s'est réuni ... pour discuter des candidatures ministérielles ... Après un bref débat, le cholx s'est porté sur les citojens Vandervelde, Wauters, Anseele, Huysmans et Laboulle" .

I'Intervention des organes des partis dans la désignation des ministres n'est évidemment pas toujours aussi flagrante. Il semble que, surtout après I945, si l'intervention des organes des partis, congrès, conseils généraux, comités centraux, etc., est constatée en ce qui concerne les programmes et le principe même de la participation gouvernementale, les questions de personne relèvent en fait de comités plus restreints et plus secrets.

( à suivro )

F. PERIN.

(I) Surtout au sein du parti catholique.

(2) Il est lui-même propriétaire et dirigeant d'une importante exploitation agricole.

( 3 ) M. Vermeylen, avocat et professeur à I'U.I.B., dirige une importante revue de droit communal i' " Ancyclopédie de jurisprudence en matière d'affaires comunales. Edition U.G.A." . 